\title{
Effects of uncertainty on melodic information processing
}

\author{
ANNABEL J. COHEN, SANDRA E. TREHUB, and LEIGH A. THORPE \\ University of Toronto, Erindale Campus, Mississauga, Ontario, Canada
}

\begin{abstract}
In three experiments, musically trained and untrained adults listened to three repetitions of a 5-note melodic sequence followed by a final melody with either the same tune as those preceding it or differing in one position by one semitone. In Experiment 1, ability to recognize the final sequence was examined as a function of redundancy at the levels of musical structure in a sequence, contour complexity of transpositions in a trial, and trial context in a session. Within a sequence, tones were related as the major or augmented triad; within a trial, the four sequences began on successively higher notes (simple macrocontour) or on randomly selected notes (complex macrocontour); and within a session, trials were either blocked (all major or all augmented) or mixed (major and augmented randomly selected). Performance was superior for major melodies, for systematic transpositions within a trial (simple macrocontours), for blocked trials, and for musically trained listeners. In Experiment 2, we examined further the effect of macrocontour. Performance on simple macrocontours exceeded that on complex, and excluded the possibil. ity that repetition of the 20-note sequences provided the entire benefit of systematic transposition in Experiment 1. The effect of musical structure (major/augmented) was also replicated. In Experiment 3, listeners provided structure ratings of ascending 20-note sequences from Experiment 2. Ratings on same trials were higher than those on corresponding different trials, in contrast to performance scores for augmented same and different trials in previous experiments. The concept of functional uncertainty was proposed to account for recognition difficulties on augmented same trials. The significant effects of redundancy on all the levels examined confirm the utility of the information-processing framework for the study of melodic sequence perception.
\end{abstract}

In the present investigation, we examine the effect of uncertainty on melodic pattern processing. In particular, we aim to show that uncertainty at various levels of stimulus structure affects melodic processing. Interest in uncertainty originated in applications of information theory to perception and meaning (Garner, 1962, 1974; Haber, 1969; Underwood, 1979). According to this conceptualization, limited capacity or processing resources can be overcome by effective coding at various stages from sensation to perception and representation.

The accuracy of encoding is thought to be affected by the physical properties of the stimulus and the experimental context. Because information requiring a simple code demands fewer processing resources than does information requiring a more complex code, complexity of the code predicts the accuracy of representation. According to Simon (1972), the complexity of a pattern can be defined by the number of symbols required to represent it. For example, a pattern with three identical elements (e.g.,

This research was supported by grants from the Natural Sciences and Engineering Research Council of Canada and from the University of Toronto. The authors thank Marilyn Barras and Donna Laxdal for their assistance in data collection. The results of Experiment 1 were reported at the International Congress on Acoustics, August 1986, Toronto. Leigh Thorpe is now with Bell Northern Research, Ltd., Ottawa, Ontario. Requests for reprints should be sent to Annabel J. Cohen, Department of Psychology, Dalhousie University, Halifax, Nova Scotia B3H 4J1, Canada. aaa) has a simpler code than one with three different elements (e.g., $a b c$ ), the former being more readily represented, remembered, and differentiated from other patterns. A similar concept underlies the "structural information theory" of Buffart and Leeuwenberg (1983), which has not been applied to music. Deutsch and Feroe (1981) proposed a hierarchical system for the representation of pitch sequences in tonal music. They argued that tone sequences redundant at different structural levels of the hierarchy would be remembered best, but they provided little empirical support (see also West, Howell, \& Cross, 1985).

The information-processing approach has been related to music perception (e.g., by Dowling \& Harwood, 1986), primarily for purposes other than prediction. Moles (1958/1968) described music in terms of information theory, measuring the uncertainty of different musical intervals and the relative frequency in the works of various composers (chap. 1), relating such measures to musical originality. Berlyne $(1971,1972)$ explored the relation between uncertainty and preference in his work on experimental aesthetics. Moreover, Chamberlain (1974) observed detrimental effects of high uncertainty on the recognition of short sequences of tones. These investigations considered uncertainty at one level of stimulus structure. In the present research, we used ordinal measures of uncertainty at various levels or time spans to investigate listeners' perceptions of tone sequences. 
There are potential advantages to examining the effects of uncertainty at various levels. First, predictions of performance based on multiple levels should be more accurate than those based on a single level. Second, results of different experiments in musical research could be related in terms of their respective manipulations of uncertainty. Third, research on music could be related to research on nonmusical patterns if both types of pattern were defined in terms of uncertainty.

We examined the effect of uncertainty on melodic discrimination at three levels: tonal or musical structure, macrocontour, and trial context. According to the information-processing view, performance should improve with reductions in uncertainty.

\section{Tonal or Musical Structure}

Generally, only a subset of tones from the diatonic (major or minor) scale is used in a melody, and within the subset, some tones are more frequent than others (Krumhansl, 1983; Moles, 1958/1968). The succession of tones is governed by certain constraints, such as the prevalence of small jumps or intervals over large, and the succession of tones in ways that imply particular chord progressions, the underlying grammar of the music (Cuddy, Cohen, \& Mewhort, 1981; Krumhansl \& Kessler, 1982).

Cuddy et al. (1981) defined a prototypical musical structure of seven tones and created musical sequences of decreasing structure by systematically degrading the basic pattern. Recognition performance decreased dramatically with decreasing structure. Moreover, ratings of perceived structure correlated highly with performance and with degree of redundancy of the stimulus. Cohen (1982), comparing performance on one sequence based on the well-structured prototype, and on another that violated the rules of the prototype, found similar effects of musical structure for transposed and untransposed comparison sequences.

Trehub, Cohen, Thorpe, and Morrongiello (1986) tested children with simpler patterns than those employed previously with adults. Their prototype outlined only one chord, the major triad (do mi sol mi do), and their untransposed comparison sequences raised a single tone by one semitone. The major triad is the most common or redundant triad structure in Western music. Redundancy, as defined by autocorrelation of the component frequencies, is also greatest. For example, frequencies of the tones comprising the major triad are related as $4: 5: 6$, but those of all other triads composed of intervals of approximately the same size are related by higher integers. These children were also tested on standards based on the augmented triad, (e.g., C E G\#), which violates the good form of the major triad (e.g., C E G). Unlike the major triad, the augmented does not imply a tonal center and has a less stable representation (Krumhansl, Bharucha, \& Kessler, 1982; Roberts \& Shaw, 1984).

The outcome was that the children's performance on the highly structured sequence, the major triad, exceeded that on the less well structured sequence, the augmented triad. In the present study, sequences based on the major and augmented triad were exemplars of high and low musically structural uncertainty.

\section{Macrocontour}

The pattern of changes in frequency direction (ups and downs) in a melody is called the contour; it is an important aspect of the information perceived in music (Dowling \& Fujitani, 1971). Child and adult listeners confuse short musical sequences that have similar contours but that differ in actual interval relations (Dowling, 1982; Morrongiello, Trehub, Thorpe, \& Capodilupo, 1985).

A number of investigators have examined the relative ease of encoding contours of different complexity, as measured by the number of frequency-direction changes of contour. Typically, superior performance is obtained for unidirectional versus bi- or multidirectional contours for sequences of 3 or 4 tones (Cuddy \& Cohen, 1976; Divenyi \& Hirsh, 1974; Nickerson \& Freeman, 1974). These effects are consistent with the informationprocessing view that increased uncertainty of contour exploits limited processing resources, leaving fewer resources for the task at hand. Note that, in such studies, the task itself does not rely on the detection of a contour change; nevertheless, the contour complexity of the stimulus influences performance.

One aspect of contour that has not been examined is higher order structure, which we designate as macrocontour. It is especially applicable to the case of a long sequence formed from a number of transposed subsequences or kernel melodies-that is, the overall frequency directional pattern of the transpositions. A simple macrocontour results when successive transpositions are in the same direction-for example, always higher than the previous melody-whereas more complex macrocontours result when successive transpositions are sometimes higher and sometimes lower than the preceding kernel. Deutsch (1980) illustrated the advantage of hierarchical encoding for memory of musical passages. She presented musically trained listeners with sequences of 12 tones and required them to write the musical notation for each sequence. For half of the sequences, a 3-note kernel was presented four times, each presentation beginning on a different note; the remaining sequences were not hierarchically structured. In the hierarchically structured case, listeners recalled $94 \%$ of the tones in their correct serial position, compared with only $52 \%$ in the unstructured case. The structured sequences, when considered at the lowest level, (i.e., that of relations within the kernel) had more contour changes than the unstructured sequences. At the higher level of the relation between transposed kernels, performance correlated with macrocontour complexity, although Deutsch (1980) did not refer to the role of contour.

We are suggesting, then, that subjective contour complexity may be reduced by systematic transposition of kernels (e.g., consistently ascending). In the present study, 
we examined the effect of systematic as opposed to random transposition, which can be understood as a macrocontour manipulation. Within the information-processing framework, systematic presentations involve less uncertainty and lower demands on processing resources, which should lead to higher performance.

\section{Task Structure}

Redundancies in experimental tasks are also thought to affect performance. For example, intensity discrimination is poorer when the standard intensity varies from trial to trial (roving standard) than when it is fixed (e.g., as reviewed by Macmillan, 1987). This difference in performance is not attributable to sensory factors, but rather to task-related constraints on contextual memory. Related effects of fixed as opposed to variable standards have been shown for consonant and vowel discrimination (Macmillan, Braida, \& Goldberg, 1987) as well as frequency discrimination (Watson \& Foyle, 1985). Similarly, Kidd, Boltz, and Jones (1984) have observed that irrelevant variation in rhythm within and between trials decreased performance in a melodic judgment task. These effects of task structure are consistent with the information-theoretical assumption that processing is facilitated by conditions of low uncertainty and high redundancy (Garner, 1974).

\section{Manipulation of Redundancy in the Present Study}

To examine the applicability of an informationtheoretical approach to melody perception, we manipulated redundancy on three different levels, each one with a different time span. Within the shortest time span, we used two melodies differing in degree of uncertainty: the major triad and augmented triad sequences previously employed by Trehub et al. (1986). At the intermediate level, we transposed sequences within a trial in regular or irregular patterns. This can be understood as a macrocontour manipulation in which successive sequences repeat the preceding contour according to a single rule (e.g., always ascend) or do so in a randomly determined way that allows successive contours to begin on notes higher or lower than the previous pattern. Finally, with a longer time span still, sequences were either blocked (only one type of melody) or mixed (with both melodies) within a session. In the latter case, the listener would be uncertain about the melody to be tested on each trial.

Uncertainty can be altered by features of the stimulus or task, as noted above. It can also be altered by characteristics of the listener such as musical experience. Musical training, by enabling higher level processing or by providing useful schemata, reduces the subjective uncertainty of stimuli. Indeed, such effects of training on music judgment have been well documented (Beal, 1985; Cuddy \& Cohen, 1976; Cuddy et al., 1981; Cuddy, Cohen, \& Miller, 1979; Krumhansl \& Shepard, 1979; Russell, 1987; Smith, 1985; Watkins, 1985).

\section{EXPERIMENT 1}

In the present experiment, we varied redundancy at the levels of sequence, trial, and session, with the expectation that performance would be related to the relative redundancy in the stimulus and the stimulus context. We also predicted superior performance for listeners with greater musical training.

\section{Method}

\section{Subjects}

The participants were 24 adults ( 11 females, 13 males) from the university community, with a mean age of 21.5 years (range of 18 to 28 years). One half of the subjects had musical experience at least equivalent to that of the Grade VIII examination of the Toronto Royal Conservatory of Music. The remaining subjects had received no more than 4 years of music training in childhood and were not currently involved in any musical activities. The subjects received token payment for their participation in three sessions.

\section{Apparatus}

Sine-tone stimuli were generated on-line by a synthesizer/function generator (Hewlett-Packard 3325A) and were presented via one channel of a stereo amplifier (Marantz, Model 1010) over a loudspeaker (Canton, xi 270). Testing was carried out inside a double-walled, sound-attenuating chamber (Industrial Acoustics Co.). A microcomputer (Commodore PET, Model 2001-16N) connected to a custom-built interface controlled the synthesizer and monitored the two keys of a small control box (two push buttons mounted on a Hammond chassis) with which the experimenter initiated trials and recorded responses from inside the testing booth. The computer also controlled the illumination and activation of toys in a Plexiglas display box within the booth.

\section{Stimuli}

The two standard sequences of 5 notes were the ascendingdescending major triad and augmented triad. Each trial consisted of four sequences: three repetitions of the standard melody and a final test sequence that either was the same melody as the standard (control trial) or differed in the third serial position by one raised semitone (experimental trial), as shown in Figure 1. Limitation of the error to 1 note and position provided additional control. For the purpose of comparison with earlier data of Trehub et al. (1986), the raised semitone change was selected for both standards.

The tones ranged in frequency from $123 \mathrm{~Hz}\left(\mathrm{~B}_{2}\right)$ to $659 \mathrm{~Hz}\left(\mathrm{E}_{5}\right)$ in equal tempered tuning (Backus, 1969). They were $200 \mathrm{msec}$ in duration, and intertone intervals were $200 \mathrm{msec}$; thus, each sequence was $1.8 \mathrm{sec}$. The intermelody interval within a trial was $800 \mathrm{msec}$. The response interval was $4 \mathrm{sec}$ from the onset of the third serial position in the final sequence of the trial.

Each session consisted of a training and test phase. For experimental trials in the test phase, the contrasting melody was formed for each standard by increasing the frequency of the middle note of the standard by 1 semitone. For control trials, the four repetitions of the standard within the trial were transposed in a way that reduced repetition of frequencies across sequences, a potential source of extraneous redundancy. Because four different augmented triads can be generated from the 12-note chromatic scale (see Figure la and c), each trial of the augmented condition of the experiment could contain four different augmented triads without repeating any frequencies. Each trial was, therefore, composed by selecting the four triad groups without replacement in one of two ways that controlled for degree of uncertainty of transpositions within a trial. 

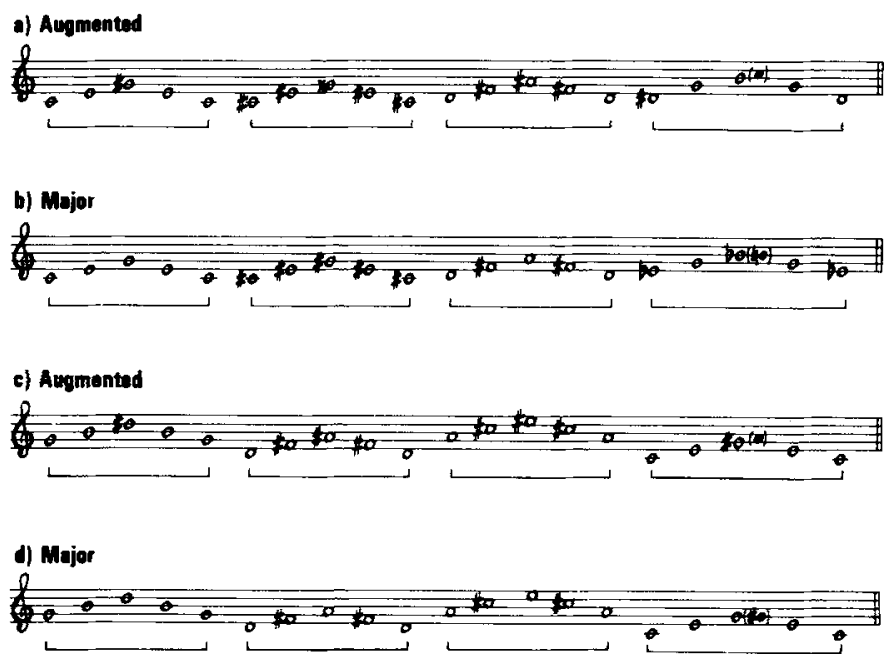

Figure 1. Stimulus sequences: (a) augmented step, (b) major step, (c) augmented random, (d) major random. The solid note of the final sequences indicates the frequency of the changed note in the incorrect comparison sequences.

For the low uncertainty or step condition of transposition uncertainty, the transpositions followed the rule that successive transpositions began 1 semitone higher than the preceding one. This is shown musically in Figure 1a, and schematically in Figure 2. There were 10 such trials, each starting on a different note. Considering the trial as a 20-note sequence, there were always seven directional changes but the macrocontour had no change of direction. For the high uncertainty or random condition, the triad group and starting note ( 1 of 3 notes) for each of the four triad groups were randomly selected (see Figures $1 \mathrm{c}$ and 2). There were 10 such trials. In the 20-note sequence, there were always seven changes in direction, and the relations among transpositions led to a macrocontour with one or two changes in direction-that is, a more complex macrocontour than in the step condition. For both the step and the random conditions of uncertainty, the contrasting melodies for experimental trials were created by altering the third serial position of the final sequence in the trial (i.e., the 18 th of the 20 notes in the trial).

The conditions with augmented standards were created as described above. For the major triad condition of both levels of uncertainty, the augmented fifth of each triad (third serial position)

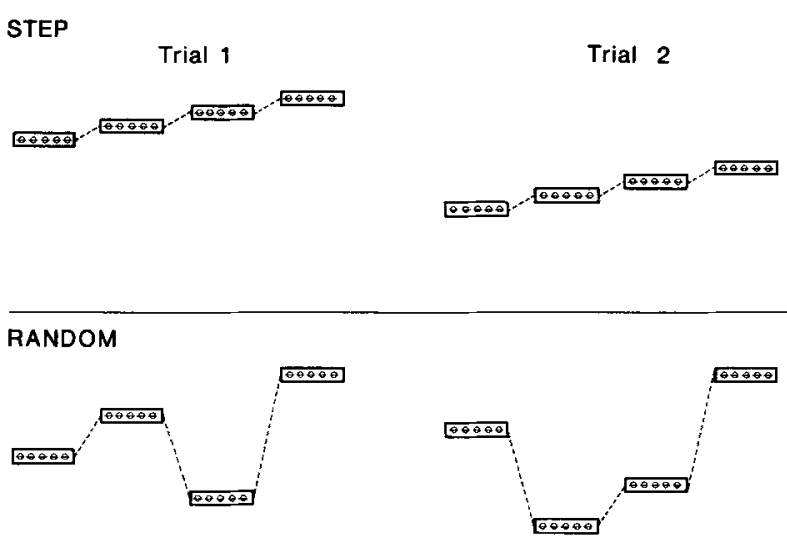

Figure 2. Schematic representation of step and random transposition condition illustrating the macrocontour manipulation. of the corresponding augmented condition was lowered to form the perfect fifth (see Figures $1 \mathrm{~b}$ and d). Thus, for each augmented trial, there was a corresponding major trial that differed only in the frequency of the third note of each sequence within the trial.

\section{Procedure}

Subjects were assigned to either the random or the step transposition condition. On each of 3 days, which were separated by approximately 1-week intervals, each subject received one of the three conditions (blocked major, blocked augmented, or mixed major/augmented). There were six possible orders of the three conditions, and within each level of training, each subject was randomly assigned to one of these orders.

Training. On each day of testing, there was a training period followed by two sessions of trials in the same condition. The subject and experimenter sat at opposite corners of the sound-attenuated room. In the first training session, the experimenter explained that the melody would be repeated three times, followed by another repetition or by a slightly different melody. The subject was to indicate whether the same or different melody occurred, and the experimenter pressed the appropriate button to record the response.

The experimental trials in the training phase of the step condition involved three contrasting melodies that differed from the standard by 1,4 , or 6 semitones in the third serial position. Similarly, the three contrasting melodies for the training phase of the random condition included contrasts of 1 and 4 semitones, and a contrast that altered the contour of the melody. The three contrasts were chosen to reflect differential ease of discrimination from the standard. Because of the difficulty of the random condition, the added cue of contour change was included to facilitate discrimination.

The experimenter initiated 2 sample experimental (change) trials followed by a sample control (no-change) trial, providing correct labels as each trial was presented. For all trials in which the listener responded correctly, a toy animal was illuminated and activated in a Plexiglas display box (following Trehub et al., 1986). Further control and experimental trials were presented in random order without the aid of the experimenter. The level of difficulty of the training trials was contingent on the performance, with difficulty increasing when four successive responses were correct and decreasing when four successive responses were incorrect. Training terminated when the subject achieved 4 correct trials in succession at the highest level of difficulty (1-semitone change) or after 23 
trials, after which the subject proceeded immediately to the test phase.

Testing. For the blocked condition, one half of the 20 trials were control (no-change) trials; the other half had a 1-semitone change in the third serial position of the final sequence. There were two sets of 20 trials, separated by a short break, making for a total of 40 test trials per day. The trials were randomly ordered within sessions, with the constraint that no more than 2 control trials would be presented successively. For the random condition, each of the 10 precomposed trials was presented twice in each session of 20 trials. In each of two sessions of 20 trials in the mixed condition, half had major standards and half had augmented standards, with equal numbers of same and different trials for each standard.

\section{Results and Discussion}

For each subject, the mean proportion correct for each condition was entered into an analysis of variance. There were four within-subjects factors: session (first/second), blocking (blocked/mixed), musical structure (major/ augmented), and trial type (control-same/experimentaldifferent). Session was included as a factor to reveal learning or fatigue effects. Experimental and control trials were compared because of different strategies that might be applied for same as opposed to different trials. There were two between-groups factors of training (trained/untrained) and pattern of transposition (step or random).

\section{Musical Structure}

Collapsing over all the variables except musical structure, performance on the major triad standard was .85 and for the augmented triad standard, .76 $[F(1,20)=$ $18.3, p<.001]$, directly replicating the effect observed by Trehub et al. (1986) for preschool children, and by Cohen (1982) for adults tested with two sequences differing in degree of musical structure. Musical structure interacted with trial type [i.e., whether test stimuli were the same as, or different from, the standard: $F(1,20)=5.20$, $p<.03$; see Figure 3].

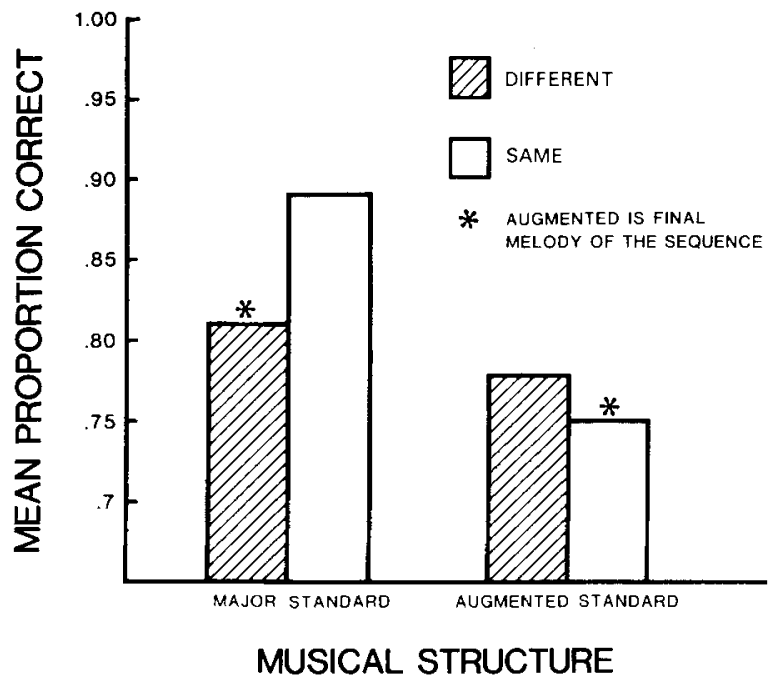

Figure 3. Mean performance for major and augmented standards for same and different trials.
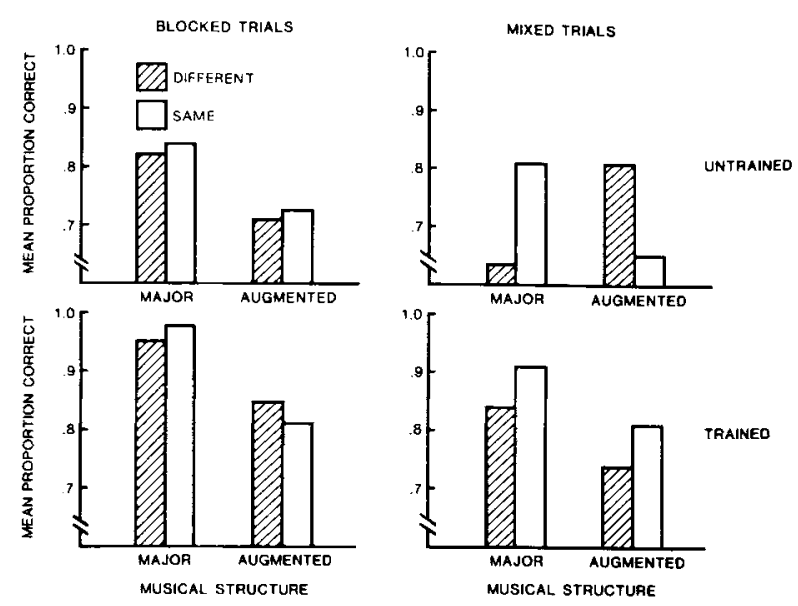

Figure 4. Mean performance as a function of musical structure (major/augmented), trial type (same/different), session context (blocked/mixed trials), and level of training.

The highest performance occurred when the major triad was unchanged. For both standards, performance was lowest when the augmented sequence was presented last in the trial. Low performance may have resulted from uncertainty arising from the two roles played by the augmented triad: incorrect sequence for the major standard, and correct sequence for the augmented standard. This could apply only in mixed blocks. When the two standards were tested (i.e., in mixed blocks) and the final sequence was augmented, it was not sufficient to categorize the sequence as augmented; it was also necessary to remember the context. If the augmented sequence was heard immediately before, then the correct response was same; otherwise, the correct response was different.

\section{Blocked versus Mixed Trials}

Evidence that untrained listeners adopted such a strategy is suggested by the interaction that included blocking and musical training in addition to musical structure and trial type $[F(1,20)=5.7, p<.026$; see Figure 4]. The effect was confined to untrained listeners on mixed trials (top right panel). Here, untrained listeners had great difficulty discriminating the augmented comparison from either standard. It is likely that untrained listeners in the mixed condition recognized the augmented sequence, but that they had difficulty recalling when they had last heard it. Such difficulty does not occur when processing is more efficient, as in the case of trained listeners, or when uncertainty is reduced by blocking trials. Collapsing over all other variables, at the highest level of structure examined, the uncertainty created by testing both sequence types in the same session decreased performance [.77 vs. $.84 ; F(1,20)=6.1, p<.02]$.

\section{Regular versus Irregular Pattern of Transposition}

A pattern of irregular (random) as opposed to regular (step) transpositions within a sequence decreased perfor- 

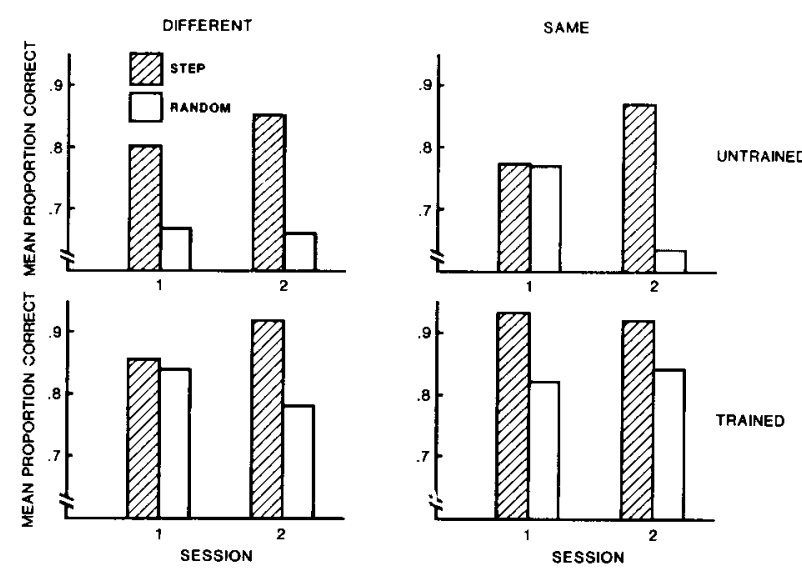

Figure 5. Mean performance as a function of session, step and random transposition, trial type (same/different), and level of training.

mance $[.75$ vs. $.86 ; F(1,20)=10.6, p<.005]$. This difference became more pronounced in the second session, when performance on the random transpositions decreased further and performance on the regular transpositions increased $[F(1,20)=10.1, p<.005]$. The effects of macrocontour pattern and session interacted with trial type and experience $[F(1,20)=11.01, p<.005$; see Figure 5]. In Session 2, this effect was most pronounced for the less trained subjects on same trials, but experience with the task was necessary before untrained listeners were affected by this superordinate level of stimulus structure.

\section{EXPERIMENT 2}

In Experiment 1, we found a superiority of regular over irregular patterns of transposition, in addition to the superiority of major over augmented sequences, and of blocking over mixed-trial presentation. Because the condition with the irregular pattern differed also in the number of distinctive 20-note melodies, increased uncertainty of melody type may have accounted for lower performance in the irregular or random macrocontour condition. In Experiment 2, we examined the effect of complex macrocontour while controlling for the effect of number of different sequences heard in a session. Accordingly, performance on a complex macrocontour sequence was compared with performance on a simple macrocontour sequence.

In Experiment 1, the irregular and regular transposed sequences also differed in range. The regular transpositions generally covered a narrower range as a result of successive transpositions of one semitone. To ensure that the observed effect did not depend on range, the ranges of the irregular and regular transpositions were made comparable.

In Experiment 1, the regular sequence was ascending. As a result, the incorrect note exceeded the original range of the sequence, providing a potential cue seldom avail- able in the irregular case. In Experiment 2, a descending sequence was employed in addition to the irregular and ascending regular sequence. The latter sequence provided a second check on the generality of the effects of macrocontour simplicity. The incorrect note in the descending sequence did not exceed the original range of the sequence; hence it did not provide an additional figural cue. Therefore, the descending sequence provided an opportunity to determine whether the absence of the figural cue would depress performance.

If discrimination and recognition depended on macrocontour complexity, then performance should be lower on the sequence with the complex macrocontour. If, however, subjects were able to benefit from hearing a 20note sequence presented repeatedly in different keys throughout the session, then no difference would be expected as a function of the internal transposition pattern. If exceeding the range by an incorrect note provided a discriminative cue, then performance on the ascending macrocontour would be expected to be higher than on the descending sequence. Were lower performance to result for the descending sequence, however, it would not be possible to exclude the interpretation that descending sequences are simply more difficult to encode and retain than ascending sequences. Cuddy and Cohen (1976) reported higher recognition performance for ascending three-note sequences based on major triads.

In addition to the examination of macrocontour complexity, the experiment provided a second opportunity to explore the effect of the augmented triad in "correct" and "incorrect" contexts.

\section{Method}

\section{Subjects}

The participants were 24 adults ( 18 fermales, 6 males) from the university community, with a mean age of 21.2 years (range of 18 to 30 years). One half had musical experience at least equivalent to that of the Grade VIII examination of the Toronto Royal Conservatory of Music (mean years of training on a major instrument was 9.1 years). The remaining subjects had no more than 4 years of musical training, and they were not currently involved in any musical activities (mean years of musical training, 1.3 years).

\section{Apparatus}

The apparatus was the same as for Experiment 1, except that the Plexiglas display box was disconnected and the subjects did not receive feedback.

\section{Stimuli}

As before, the two standard sequences of 5 notes were the ascending-descending major triad and augmented triad. Also retained were trial structure with respect to repetitions of the standard melody within the sequence, incorrect tone in the third serial position of the final sequence, timing (i.e., tone duration, intermelody interval, and response interval), and experiment structure (three sessions, each with training and test phases).

Each subject was tested in each of three conditions, which differed in the arrangement of the transpositions within each trial (see Figure 6). In the complex condition, the pattern was selected from Experiment 1; in the step-up condition, all sequences successively ascended; in the step-down condition, all sequences successively descended. In contrast to Experiment 1 , in which successive trans- 

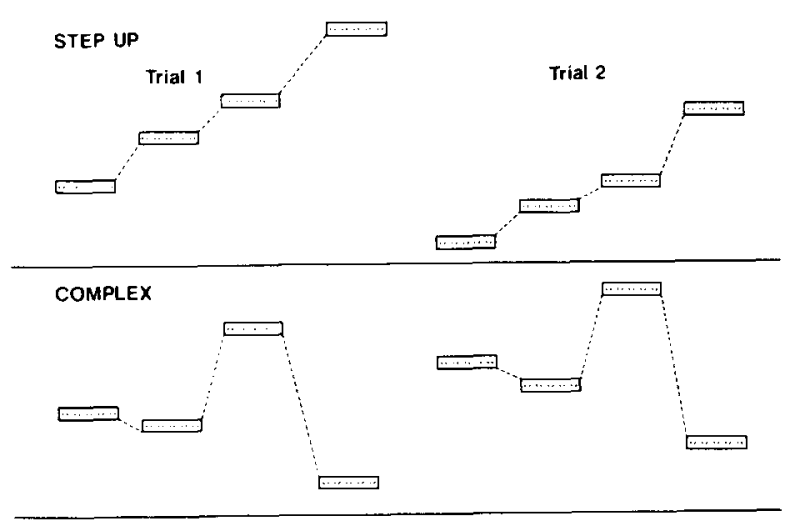

STEP DOWN

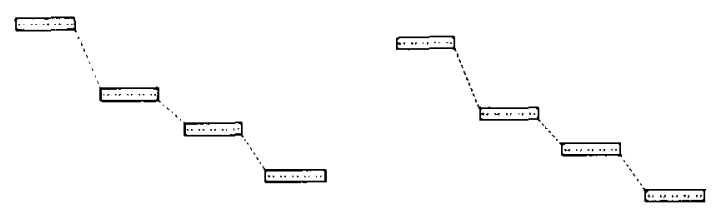

Figure 6. Trial structure for step-up, complex, and step-down conditions of transposition pattern.

positions were 1 semitone higher, transpositions in the step-up condition were 3,2 , and 5 semitones higher, respectively, and transpositions in the step-down condition were 5,2 , and 3 semitones lower, respectively (see Figure 6). In the complex condition, the transpositions were down 2 , up 7 , and down 10 semitones, respectively. The range of all three conditions of transposition was identical - from $123 \mathrm{~Hz}\left(B_{2}\right)$ to $587 \mathrm{~Hz}\left(D_{5}\right)$-and the constraint on repetition of notes within a trial was preserved, as in Experiment 1.

\section{Procedure}

On each of 3 days, which were separated by approximately 1week intervals, each listener received one of the three conditions (complex, step-up, or step-down). There were six possible orders of the three conditions and within each level of training, 2 subjects were randomly assigned to one of these orders. The training and testing procedures were the same as in Experiment 1, except for the omission of visual reinforcement. There were two sessions of 20 trials with 10 major standards and 10 augmented. For each of the two standards, half the comparisons were unchanged and the other half were changed.

\section{Results and Discussion}

For each listener, the mean proportion correct for each condition was entered into an analysis of variance. There were four within-subjects factors: session (first/second), transposition (complex, step-up, step-down), melodic structure (major/augmented), and trial type (same/different). There was one between-groups factor, training (trained/untrained).

\section{Pattern of Transposition}

Consistent with the prediction that the irregular transposition would lead to the lowest performance, mean proportion correct on the complex pattern (.73) was lower than on the step-up (.81) and step-down (.76) sequences and the effect of macrocontour pattern was highly significant $[F(2,44)=9.1, p<.001]$. The effect of macro- contour pattern was attributable primarily to different trials $[F(2,44)=11.6, p<.005$; see Figure 7]. For different trials, performance on ascending sequences $(.84)$ was significantly higher than that on complex macrocontours (.66), but it was not significantly higher than that on descending sequences $[.70$; Tukey tests, $W(6,44)=.18$, $p<.05]$.

As shown in Figure 7, the effect of macrocontour on different trials was strongest in Session 1. The difference between same and different trial types leveled off over the two sessions; the triple interaction among macrocontour, sameldifferent trial type, and session was significant $[F(2,44)=3.5, p<.05]$. The interaction of macrocontour, same/different trial type, and musical structure was also significant $[F(2,44)=3.3, p<.05]$. For both major and augmented sequences, the benefit of a regular pattern was pronounced for different trials (see Figure 8). For augmented sequences, a marked decrease in performance appeared for the step-up same condition. Recall that a similar difficulty on augmented same trials

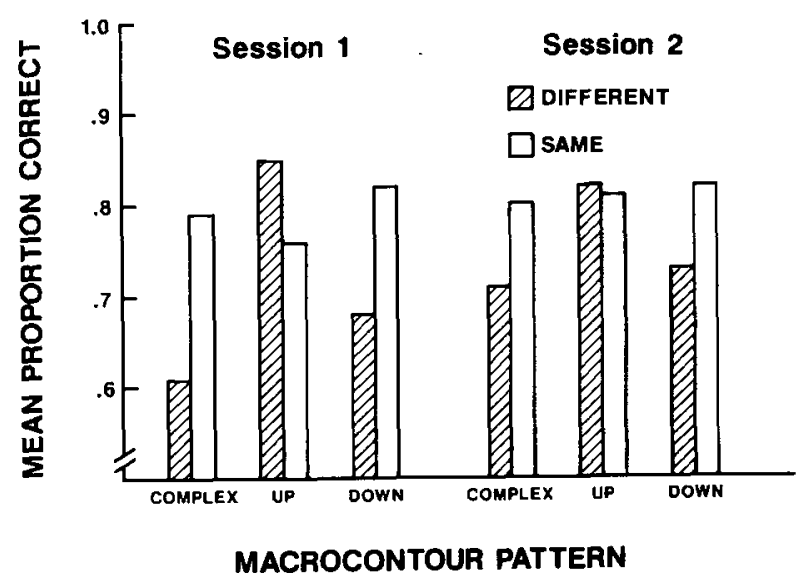

Figure 7. Mean performance as a function of transposition pattern (step-up, complex, step-down), session (first/second), and trial type (same/different).

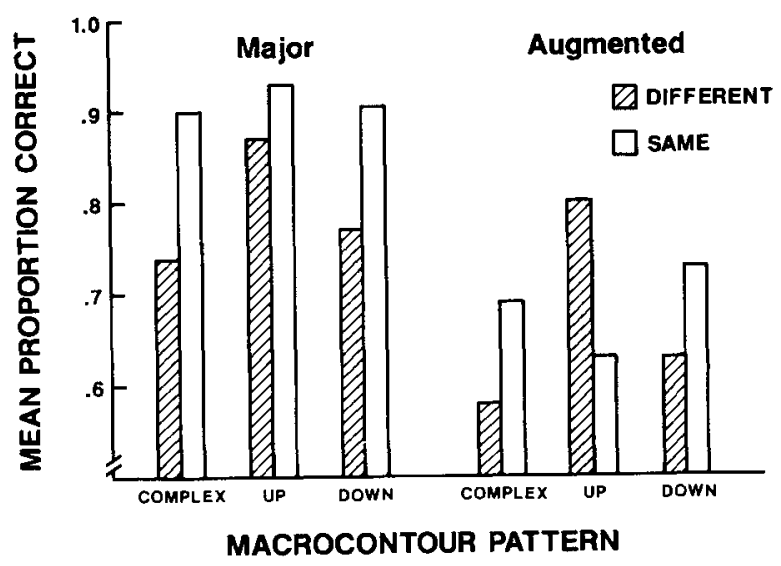

Figure 8. Mean performance as a function of transposition pattern (step-up, complex, step-down), musical structure (major/ augmented), and trial type (same/different). 
occurred in Experiment 1, which also involved an ascending condition.

\section{Musical Structure}

More generally, the superiority of major over augmented triad sequences was very strong $[F(1,22)=54.4$, $p<.0001]$, especially for experienced listeners $[F(1,22)$ $=9.1, p<.01]$. As in Experiment 1, same conditions of the major triad led to higher performance than did different conditions $[F(1,22)=4.9, p<.05]$; and the interaction of major/augmented, experimental/control, and level of training (see Figure 9) replicated the pattern of findings observed in Experiment 1 [panel 2, Figure 4; $F(1,22)=5.7, p<.025]$. Note in particular that performance on the augmented same trials did not exceed that for augmented different trials for untrained listeners.

The benefit of same trials for both major and augmented sequences was found only for the highly trained listeners. Less trained listeners did more poorly when the last sequence of the trial was augmented (experimental trials for major sequences, control trials for augmented sequences), possibly reflecting the effect of uncertainty resulting from the double function (correct or incorrect) of the augmented triad in the last position. Although such uncertainty may also create a problem for trained listeners, different strategies for coding major and augmented triads may lead to
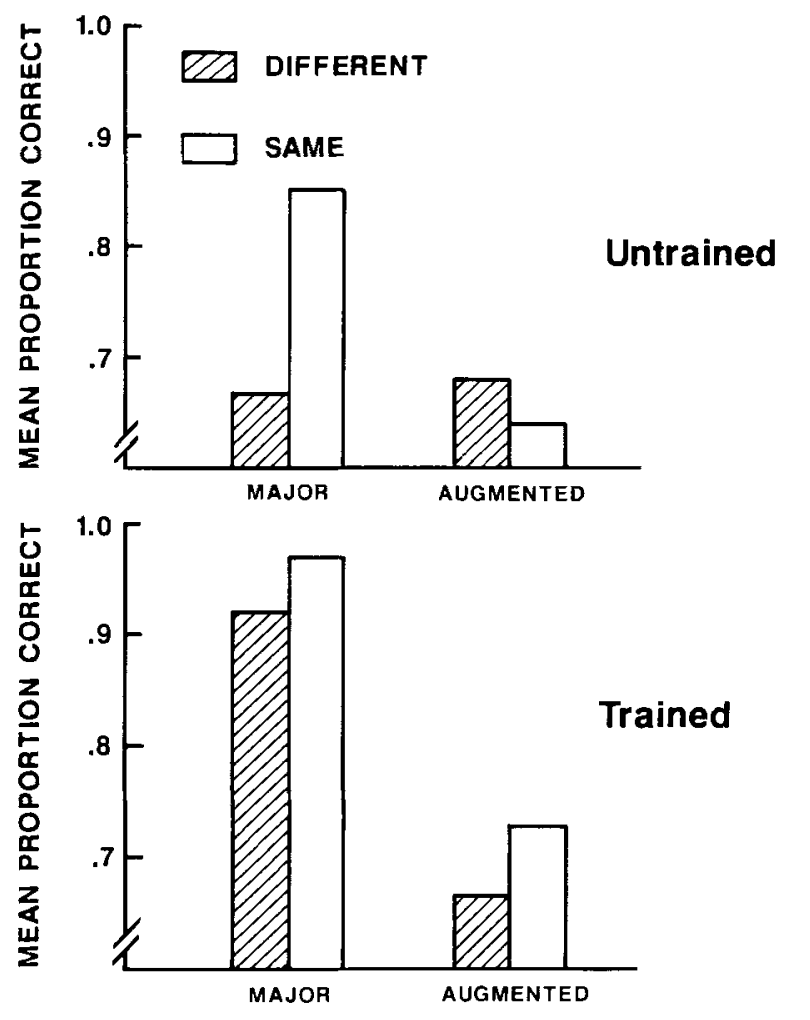

MUSICAL STRUCTURE

Figure 9. Mean performance as a function of musical structure (major/augmented), trial type (same/different), and level of training. a uniform advantage of same over different conditions, regardless of whether the sequence is major or augmented.

\section{EXPERIMENT 3}

In Experiment 1, listeners with less musical experience performed more poorly on augmented same trials than on augmented different trials. This pattern reappeared in Experiment 2. Also, for all subjects, the pattern appeared for ascending sequences in mixed blocks-though not for irregular or descending sequences. In an effort to provide additional information about the uncertainty account of poor performance on the augmented same condition, Experiment 3 was focused on the ascending condition.

It has already been suggested that the condition is difficult because of the double function of the augmented triad-as the different melody for major standards, and as the same melody for augmented standards. Recognition of the augmented triad in this position does not in itself provide sufficient information for task solution. In contrast, recognition of the major triad in the final position of the trial does allow for task solution. On the one hand, uncertainty in the augmented triad function could account for lower performance. On the other hand, if the augmented same trial sequences are encoded as more unstable structures overall than the augmented different trial sequences, then information processing for augmented same trials should be less accurate than that for augmented different trials.

In order to obtain a measure of the perceived structure of the 20-note trials, listeners were asked to provide ratings. Cuddy et al. (1981) found high correlations between ratings and recognition performance for sequences of 7 notes. In the present case, we would expect major same trials to receive the highest ratings, but the ordering of the remaining sequences was an empirical question. Of particular interest was the ordering of augmented same and different trials. If listeners aggregated the structure in each of the four sequences in a trial, then the four triads of the augmented same trials would be rated lower than the four triads of the augmented different trials, which involved a minor triad, known to be more stable than the augmented (Roberts \& Shaw, 1984). However, if listeners noticed repetition of the augmented triads in same trials, ratings could exceed those for different trials where such regularity would not be apparent.

If augmented same trials received the lowest ratings, this would suggest that uncertainty from four triads was cumulative and overrode any positive effect of simple repetition at a higher level. This account would explain the poor performance in the earlier experiments in terms of high overall instability. If ratings on the augmented same trials exceeded those for augmented different, this would suggest that repetition of the augmented sequences was detected and was more salient than detection of an "odd one out" in the augmented different trials. Given the latter outcome, it could be concluded that difficulty on the same condition in the earlier experiments resulted 
from additional uncertainty created by the perceived double function of the augmented triad ending rather than by the presence of four unstable triads.

\section{Method}

Subjects

The participants were 20 adults ( 10 females, 10 males) from the university community, with a mean age of 20.85 years (range of 19 to 24 years). Half of the subjects had musical experience at least equivalent to that of Grade VIII examination of the Royal Conservatory of Music (mean years of training on the major instrument was 8.3 years). Their mean age was 20.9 years. The remaining subjects had received no more than 4 years of music training and were not currently involved in any musical activities (mean years of musical training 1.0 years). Their mean age was 20.8 years.

\section{Apparatus}

The apparatus for generation of the stimuli was the same as in the previous experiments.

\section{Stimuli}

From the ascending condition of Experiment 2, a tape-recording (Tandberg reel-to-reel tape recorder, model $9200 \mathrm{XD}$ ) of three practice trials and two random orders of 20 test trials was produced. Response time or intertrial interval was approximately $6 \mathrm{sec}$.

\section{Procedure}

The sequences were presented to listeners in groups of 1 to 8 in a quiet room. For playback, we used a reel-to-reel Tandberg TD tape recorder, Realistic SA-10 amplifier, and Radio Shack Nova-6 loudspeakers.

The following instructions were given to the subjects:

This experiment concerns the perception of structure in musical sequences. You will hear sequences of 20 notes and will be asked to rate how well or poorly structured each sequence sounds to you. Assign " $l$ " if the sequence is very poorly structured, and contains jarring or unexpected notes. Assign " 7 " if the sequence is very well structured and contains no jarring or unexpected notes. Intermediate numbers " 2 " and " 6 " reflect the relative degree of perceived structure in the sequences in terms of the rating scale where " $\mathrm{l}$ " is low and " 7 " is high.

There are 3 practice sequences which will give you an idea of the type of patterns you will be hearing. Please ask any questions after the practice session if the procedure is still unclear to you.

Following the practice trials, there are two sets of 20 test trials. Make your best estimate of the perceived structure for each sequence; there are, however, no right or wrong answers.

\section{Results and Discussion}

For each subject, the mean of the five ratings for each of the four stimulus sequence types (major same, major different, augmented same, augmented different), for each of the two sessions, was entered into an analysis of variance. There were three within-subject factors-musical structure (major/augmented), trial type (same/different), and session (first/second) - and one between-groups factor, musical training. As expected, major triad sequences were judged higher in structure than were augmented ones $[5.4$ vs. $3.8 ; F(1,18)=24.9, p<.0005]$, and same trials were judged higher than different trials [5.0 vs. 4.2 ; $F(1,18)=59.6, p<.0001]$.

Of special interest was the higher rating given to augmented same trials than to augmented different trials $(4.0$ vs. 3.6; see Figure 10). Although this difference was not significant in a Tukey test, there was a significant inter-

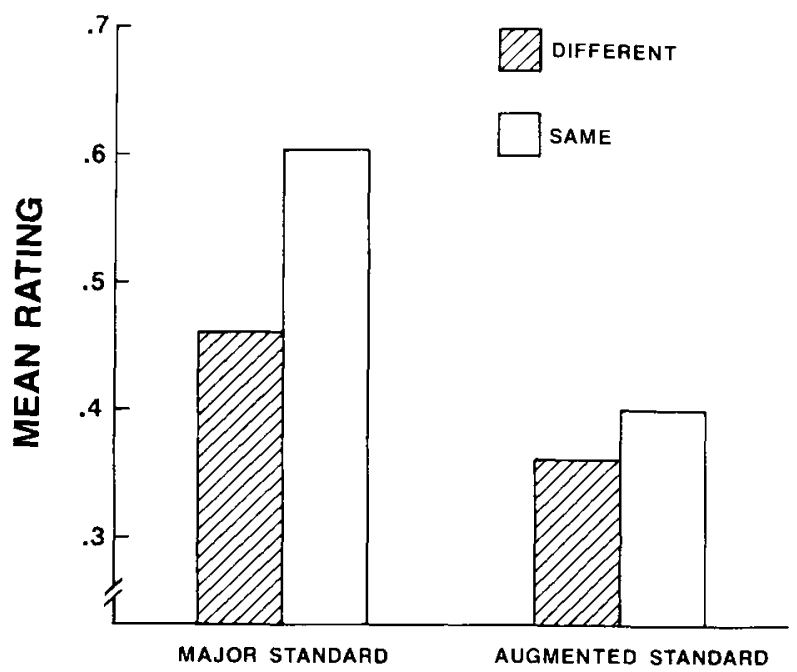

\section{MUSICAL STRUCTURE}

Figure 10. Mean structure rating for major and augmented standards for same and different trials.

action between musical structure and trial type $[F(1,18)$ $=15.0, p<.001]$. In Tukey tests, the major same score was significantly higher than all other conditions, and the major different score was significantly higher than the augmented different and augmented same scores $[W(4,24)=$ $.67, p<.05]$. There were no effects of musical training.

These findings imply that the processing disadvantage of augmented same trials in recognition tasks does not result from the overall instability of four augmented triads, but rather from the uncertainty added by the double function of the augmented triad in both major different and augmented same trials.

\section{GENERAL DISCUSSION}

In Experiments 1 and 2, we examined the effects of uncertainty at various levels of musical structure on melodic discrimination and recognition. The main effects of musical structure, training, pattern of transposition, or macrocontour, as well as of blocking of trials, are consistent with ordinal predictions from the information-processing approach. The findings are also consistent with predictions about the cumulative effects of changes. For example, high performance (.98 proportion correct in Experiment 1) was associated with the major standard, blocked condition, regular pattern of transposition, and highly trained subjects. In contrast, low performance (.68) was associated with the augmented standard, mixed condition, irregular pattern of transposition, and untrained listeners. Interactions among musical structure, pattern of transposition, blocking, and training indicate the interdependence of these effects.

In Experiments 1 and 2, the significant decrease in performance by random transposition is predictable from a 
macrocontour complexity rule. If this were the only explanation, then trials with higher macrocontour complexity should have produced the lowest performance. This was not always the case. Performance on individual trials ranged from .69 to .85 , and both extremes were associated with two directional changes of macrocontour. It is possible that the size of the transpositions and the frequency range of the sequences played a role. These interpretive problems may stem from the conventional definition of contour, which considers the pattern of directional change in frequency (Dowling \& Fujitani, 1971) but ignores the magnitude of frequency change between successive tones and the overall frequency range of the melody. This conceptualization of contour may be appropriate for listeners with little musical experience, such as infants (Trehub, Bull, \& Thorpe, 1984) or naive adult listeners (Friedman, 1985), but it may be less appropriate for experienced listeners.

Trained listeners may achieve superior performance by using nominal codes (e.g., major or augmented) that are unavailable to less trained listeners. With or without such codes, trained listeners may encode musical material at a higher level, and as a result, experience reduced uncertainty with well-structured stimuli.

Our results extend the findings of Kidd et al. (1984), who showed that increasing rhythmic uncertainty on a number of levels led to increased errors in the identification of a melodic change. It is clear that the effects of uncertainty are not confined to temporal factors that were constant in the present study. Such uncertainty may depend upon structure particular to music (e.g., major vs. augmented), relations in the music that can be described by simple rules of transposition (cf. Restle, 1971), and structure of the task (Kidd et al., 1984)-as in the case of mixed and blocked trials and the double role of the augmented triad in final position, as discussed in Experiment 3.

The findings place in perspective effects of musical structure as opposed to task structure. Paradigmatic manipulations such as the inclusion of mixed and blocked trials are not specific to musical stimuli. Similarly, contour manipulations are not limited to music. For example, the ordering of items in a visual display can be conceptualized in terms of simple and complex contour. In contrast, the manipulation of major and augmented chord structure is specific to the musical domain. Nevertheless, all of the aforementioned manipulations had effects on performance, and such manipulations, musical and nonmusical, can be described in terms of uncertainty. Thus, our findings support the viability of the information-processing approach to research in music perception.

Finally, the idea of considering various levels of uncertainty simultaneously is also applicable to the study of musical aesthetics. The present study provides insight into the perceived similarity among variations in music and, in this sense, extends previous research on the relation between musical appreciation and uncertainty (Berlyne, 1971).

\section{REFERENCES}

Backus, J. (1969). The acoustical foundations of music. New York: Norton.

BEAL, A. L. (1985). The skill of recognizing musical structures. Memory \& Cognition, 13, 405-412.

BERLYNE, D. (1971). Aesthetics and psychobiology. New York: Wiley. BERLYNE, D. (1972). Affective aspects of aesthetic communication. In T. Alloway, L. Krames, \& P. Pliner (Eds.), Communication and affect: A comparative approach (pp. 97-118). New York: Academic Press.

BuffarT, H., \& LeEuWENBERG, E. (1983). Structural information theory. In H. G. Geissler, H. F. J. M. Buffart, E. L. J. Leeuwenberg, \& V. Sarris (Eds.), Modern issues in perception (pp. 48-72). New York: North Holland.

Chamberlain, P. J. (1974). Pitch and duration in recognition of musiclike structures. Perceptual \& Motor Skills, 38, 419-428.

Cohen, A. J. (1982). Exploring the sensitivity to structure in music. Canadian University Music Review, 3, 15-30.

Cuddy, L. L., \& COHEN, A. J. (1976). Recognition of transposed melodic sequences. Quarterly Journal of Experimental Psychology, 28, 255-270

Cuddy, L. L., Cohen, A. J., \& Mewhort, D. J. K. (1981). Perception of structure in short melodic sequences. Journal of Experimental Psychology: Human Perception \& Performance, 7, 869-883.

Cuddy, L. L., \& Cohen, A. J., Miller, J. (1979). Melody recognition. Canadian Journal of Psychology, 33, 148-156.

DEUTSCH, D. (1980). The processing of structured and unstructured tonal sequences. Perception \& Psychophysics, 28, 381-389.

DeutsCh, D., \& Feroe, J. (1981). The internal representation of pitch sequences in tonal music. Psychological Review, 88, 503-522.

DiVENYI, P. L., \& HiRSH, I. J. (1974). Identification of temporal order in three-tone sequences. Journal of the Acoustical Society of America, 56, 144-151.

Dowling, W. J. (1982). Melodic information processing and its development. In D. Deutsch (Ed.), The psychology of music (pp. 413429). New York: Academic Press.

Dowling, W. J. \& FuJitani, D. (1971). Contour, interval, and pitch recognition in memory for melodies. Joumal of the Acoustical Society of America, 49, 524-531.

Dowling, W. J., \& HARWOOD, D. (1986). Music perception. Orlando, FL: Academic Press.

Friedman, M. L. (1985). A methodology for the discussion of contour: Its application to Schoenberg's music. Journal of Music Theory, 29, 223-248.

GARNER, W. R. (1962). Uncertainty and structure as psychological concepts. New York: Wiley

GARNER, W. R. (1974). The processing of information and structure New York: Wiley.

HABER, R. N. (1969). Information processing approaches to visual perception. New York: Holt, Rinehart \& Winston.

KIDD, G., BoltZ, M., \& JoNEs, M. R. (1984). Some effects of rhythmic context on melody recognition. American Journal of Psychology, 97, 153-173.

KrumhansL, C. L. (1983). Perceptual structures for tonal music. Music Perception, 1, 28-62.

Krumhansl, C. L., Bharucha, J. J., Kessler, E. J. (1982). Perceived harmonic structure of chords in three related musical keys. Journal of Experimental Psychology: Human Perception \& Performance, 8, 24-36.

Krumhansl, C. L., \& Kessler, E. J. (1982). Tracing the dynamic changes in perceived tonal organization in a spatial representation of musical keys. Psychological Review, 89, 334-368.

Krumhansl, C. L., \& SHePard, R. N. (1979). Quantification of the hierarchy of tonal functions within a diatonic context. Journal of Ex- 
perimental Psychology: Human Perception \& Performance, 5, 579-594.

Macmillan, N. A. (1987). Beyond the categorical/continuous distinction: A psychophysical approach to processing modes. In S. Harnad (Ed.), Categorical perception (pp. 53-85). New York: Cambridge University Press.

Macmillan, N. A., Braida, L. D., \& Goldberg, R. F. (1987). Central and peripheral processes in the perception of speech and nonspeech sounds. In M. E. H. Schouten (Ed.), The psychophysics of speech perception (pp. 28-45). Boston: Martinus Nijhoff (NATO-ASI series).

Moles, A. (1968). Information theory and esthetic perception. (J. E. Cohen, Trans.). Urbana: University of Ilinois Press. (Original work published 1958)

Morrongiello, B. A., Trehub, S. E., Thorpe, L. A., \& Capodilupo, S. (1985). Children's perception of melodies: The role of contour, frequency, and rate of presentation. Journal of Experimental Child Psychology, 40, 279-292.

NickERSON, R. S., \& FreEMAN, B. (1974). Discrimination of the order of the components of repeating tone sequences: Effects of frequency separation and extensive practice. Perception \& Psychophysics, 16, $471-477$

RESTLE, F. (1971). Theory of serial pattern learning: Structural trees. Psychological Review, 77, 481-495.

RoBerts, L. A., \& Shaw, M. L. (1984). Perceived structure of musical triads. Music Perception, 2, 95-124.

Russell, P. A. (1987). Memory for music: A study of musical and listener factors. British Journal of Psychology, 78, 335-347.
Simon, H. A. (1972). Complexity and the representation of patterned sequences of symbols. Psychological Review, 79, 369-382.

SмIтH, J. (1985). Expertise in rhythm processing: Musicians vs. nonmusicians. Berlin: Max Planck Institute for Human Development \& Education.

Trehub, S. E., Bull, D., \& Thorpe, L. A. (1984). Infants' perception of melodies: The role of melodic contour. Child Development, $55,821-830$.

Trehub, S. E., Cohen, A. J., Thorpe, L. A., \& Morrongiello, B. A. (1986). Development of the perception of musical relations: Semitone and diatonic structure. Journal of Experimental Psychology: $\mathbf{H u}$ man Perception \& Performance, 12, 295-301.

UNDERWOOD, G. (1979). Concepts in information processing theory. In G. Underwood (Ed.), Strategies of information processing (pp. 122). London: Academic Press.

WatKINS, A. J. (1985). Perceptual aspects of synthesized approximations to melody. Journal of the Acoustical Society of America, 78, 1177-1186.

Watson, C. S., \& Foyle, D. C. (1985). Central factors in the discrimination and identification of complex sounds. Journal of the Acoustical Society of America, 78, 375-380.

West, R., Howell, P., \& CRoss, I. (1985). Modelling perceived musical structure. In P. Howell, I. Cross, \& R. West (Eds.), Musical structure and cognition (pp. 21-52). London: Academic Press.

(Manuscript received August 12, 1988; accepted for publication November 30,1988 .) 